\title{
Mapping HIV-related behavioural surveillance among injecting drug users in Europe, 2008
}

V Hope (vivian.hope@lshtm.ac.uk) ${ }^{1}$, A Jeannin², B Spencer², J P Gervasoni², M J van de Laar ${ }^{3}$, F Dubois-Arber ${ }^{2}$, the ECDC HIV and STI Behavioural Surveillance Mapping Group ${ }^{4}$

1. Centre for Research on Drugs and Health Behaviour, London School of Hygiene and Tropical Medicine, London, United Kingdom

2. Institute for Social and Preventive Medicine (IUMSP), University of Lausanne, Lausanne, Switzerland

3. European Centre for Disease Prevention and Control (ECDC), Stockholm, Sweden

4. Members of the group are listed at the end of the article

Citation style for this article:

Hope V, Jeannin A, Spencer B, Gervasoni JP, van de Laar MJ, Dubois-Arber F, the ECDC HIV and STI Behavioural Surveillance Mapping Group. Mapping HIV-related behavioural surveillance among injecting drug users in Europe, 2008.

Euro Surveill. 2011;16(36):pii=19960. Available online: http://www.eurosurveillance.org/ViewArticle.aspx?Articleld=19960

Article published on 8 September 2011

The systematic collection of behavioural information is an important component of second-generation HIV surveillance. The extent of behavioural surveillance among injecting drug users (IDUs) in Europe was examined using data collected through a questionnaire sent to all 31 countries of the European Union and European Free Trade Association as part of a European-wide behavioural surveillance mapping study on HIV and other sexually transmitted infections. The questionnaire was returned by 28 countries during August to September 2008: 16 reported behavioural surveillance studies (two provided no further details). A total of 12 countries used repeated surveys for behavioural surveillance and five used their Treatment Demand Indicator system (three used both approaches). The data collected focused on drug use, injecting practices, testing for HIV and hepatitis C virus and access to healthcare. Eight countries had set national indicators: three indicators were each reported by five countries: the sharing any injecting equipment, uptake of HIV testing and uptake of hepatitis C virus testing. The recall periods used varied. Seven countries reported conducting one-off behavioural surveys (in one country without a repeated survey, these resulted an informal surveillance structure). All countries used convenience sampling, with service-based recruitment being the most common approach. Four countries had used respondent-driven sampling. Three fifths of the countries responding $(18 / 28)$ reported behavioural surveillance activities among IDUs; however, harmonisation of behavioural surveillance indicators is needed.

\section{Introduction}

Injecting drug users (IDUs) are vulnerable to a wide range of viral and bacterial infections through poor injection hygiene [1-3]. These infections, which include HIV, hepatitis C and hepatitis B, result in considerable levels of morbidity and mortality. With an estimated 750,000 to 1 million active IDUs in the European Union (EU) [4], these infections have the potential to place a considerable burden on European healthcare systems, as well as adversely impacting on the well-being of those who inject drugs.

Interventions have been adopted throughout Europe that aim to reduce risk of these infections [5]; these interventions include opiate substitution therapy (OST) and needle and syringe exchange programmes (NSPs), both of which have been shown to effective in preventing infections [6-10]. They aim to reduce infections by changing the behaviours that place individuals at risk of infection, such as through reducing the sharing and reuse of injecting equipment and by decreasing the frequency of drug injection. Monitoring the levels of these behaviours is thus important for assessing the impact of intervention programmes [11]. The systematic collection of information on risk and protective behaviours is therefore an important part of second-generation HIV surveillance systems [12]. Behavioural surveillance focused on IDUs often looks at behaviours related to a range of viral infections of the blood, not just HIV, due to the similarities in the routes of transmission [13].

In response to the HIV epidemic, some countries in Europe established studies to monitor HIV and/ or related risk behaviours among IDUs $[14,15]$. The high burden due to infections among IDUs resulted in the European Monitoring Centre for Drugs and Drug Addiction (EMCCDA) developing its drug-related infectious disease key indicator [13]. This indicator has collected data on the prevalence of HIV and hepatitis B and $C$ since the late 1990 s, and more recently has collated behavioural data.

We examine here the extent of behavioural surveillance among IDUs in the EU Member States and European Free Trade Association (EFTA) countries is examined, focusing on the methods employed and the indicators used. The EU/EFTA countries are Austria, Belgium, Bulgaria, Cyprus, Czech Republic, Denmark, Estonia, Finland, France, Germany, Greece, Hungary, 
Iceland, Ireland, Italy, Latvia, Liechtenstein, Lithuania, Luxembourg, Malta, Netherlands, Norway, Poland, Portugal, Romania, Slovakia, Slovenia, Spain, Sweden, Switzerland and the United Kingdom.

\section{Methods}

During August and September 2008, a survey was undertaken of all EU Member States and EFTA countries about behavioural surveillance activities related to HIV and other sexually transmitted infections (STIs).. Each country was sent nine separate questionnaires $[16,17]$. One explored the overall national system for behavioural surveillance and second-generation HIV surveillance. The remaining eight questionnaires each asked about a specific subpopulation (general population, youth, men who have sex with men (MSM), IDUs, STI clinic attendees, migrants, sex workers and people living with HIV/AIDS). It was emphasised on each questionnaire that the focus was behavioural data collection, as opposed to biological surveillance.

The population-specific questionnaires identified whether a country had undertaken behavioural surveillance activities for that population and if so, asked them to provide information about the methodology used. In particular, more details were requested with respect to the year(s) in which behavioural studies had been performed (since 1985), sample sizes, target populations, geographical coverage, and the recruitment and data collection methods used. Information was requested on: (i) all of the repeated studies undertaken, that is, either cross-sectional behavioural surveys that have been repeated over time, cohort studies and any other repeated collections of behavioural data (referred to as 'behavioural surveillance studies'); and (ii) any one-off behavioural surveys that had been conducted, that is, surveys that have only been undertaken at a single point in time (referred to as 'one-off surveys'). Respondents were asked to indicate the main topics covered in the behavioural surveillance studies from a detailed list grouped as follows: knowledge and attitudes regarding HIV and other STIs, sexual relationships and sexual partners, sexual activity and lifestyle, exposure to risk of infection, HIV and STI testing, drugs and substance use. Information was also requested on any main indicators that the country was currently using for monitoring purposes that were based on the behavioural surveillance data.

The questionnaires were sent by email to people in the countries who were the contact points for HIV surveillance for the European Centre for Disease Prevention and Control (ECDC), with the option of consulting other colleagues with specialist knowledge to complete the questionnaires. In the case of the IDU questionnaire, the contact points were encouraged to liaise with the European Monitoring Centre for Drugs and Drug Addiction (EMCDDA) national focal point. The key contacts returned the completed questionnaires and these were loaded into a password-protected database. The data for each population were analysed separately by an expert team member (listed at the end of this article).

In February 2009, a draft mapping of behavioural surveillance activities was presented and discussed at the Behavioural Surveillance Expert Meeting that was organised as part of the project. A total of 50 participants, including experts in behavioural surveys in the various populations, national experts and representatives of international organisations - EMCDDA, the World Health Organization (WHO) and the Joint United Nations Programme on HIV/AIDS (UNAIDS) - reviewed the mapping and the suggested sets of indicators. A revised draft of the mapping was sent to the countries for validation and 11 provided additional information on there activities, which was then incorporated into the final mapping.

\section{Results}

Of the 31 countries invited to participate, 28 returned a questionnaire on IDUs. Of these 28,18 reported behavioural surveillance activities among IDUs: 16 indicated that they had one or more behavioural surveillance studies and seven had conducted one-off surveys. Five countries had conducted both types of studies. Thus 10 of the 28 responding countries reported having no behavioural surveillance related activities among IDUs.

\section{Behavioural surveillance studies}

Of the 16 countries that had conducted one or more behavioural surveillance studies among IDUs, two did not provide further details. Among the other 14 countries, either repeated surveys or cohorts were used and/or data were collected through the national Treatment Demand Indicator system. Such systems collect data on the drug use and demographic characteristics of all drug users entering into drug treatment programmes [18]. All EU Member States have such a system to collect data from the clinical assessments of those presenting for treatment, but most do not use it to collect information on risk behaviours related to HIV and other infections. Five countries reported using their national Treatment Demand Indicator system for collecting national HIV-related behavioural surveillance data (France, Ireland, Luxemburg, Slovenia and Spain) and in two, it was the only system used (Ireland and Luxembourg).

Of the 29 behavioural surveillance studies, 27 used a repeated survey and two used cohorts (Table 1); 23 studies were still ongoing. They were reported by 12 countries (Belgium, Estonia, Finland, France, Greece, Lithuania, Netherlands, Poland, Slovenia, Spain, Switzerland and United Kingdom). Among the studies, 19 used face-to-face interviewing and eight subject-completed paper questionnaires; for one, the method was stated 'other' and for one, the method was not reported (Table 1). Annual samples sizes ranged from 100 to over 3,000 (mean: 1,107; median: $400)$. The vast majority of the repeated surveys $(21 / 27)$ recruited IDUs; however, in three countries (France, 


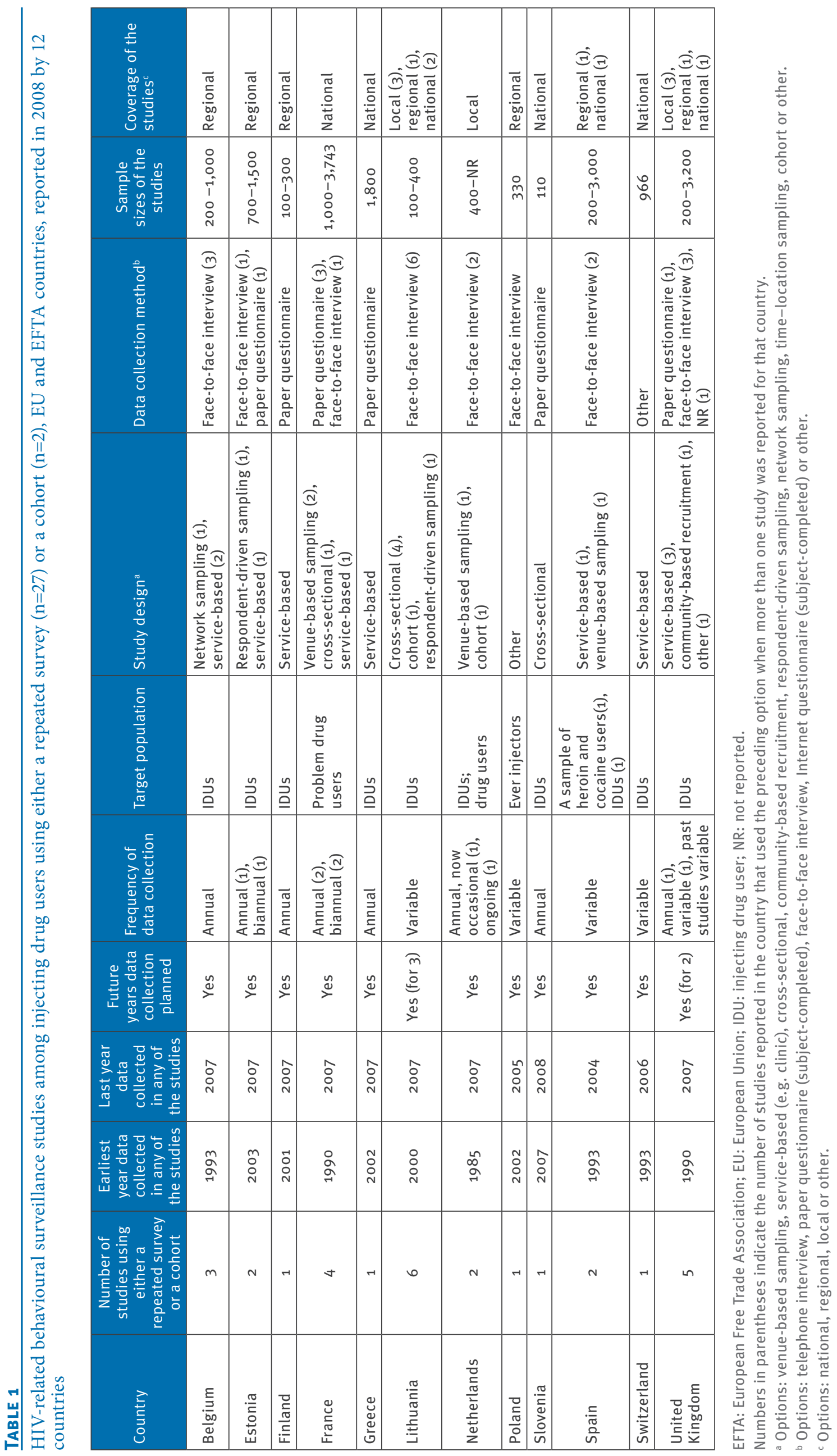


Netherlands and Spain), problem drug users (not just IDUs) were recruited. Of the 27 studies using repeated surveys, 10 had national coverage, 10 covered one region or selected regions, and seven were local. Seven countries had one or more repeated surveys with national coverage (Table 1).

Seven countries had used two or more repeated surveys or cohorts for behavioural surveillance (Belgium, Estonia, France, Lithuania, Netherlands, Spain and United Kingdom), with different geographical coverage, target populations, and/or settings used within the countries.
Topics covered by the behavioural surveillance studies

The topics covered by data collected in the behavioural surveillance studies focused on drug use, injecting practice, HIV and hepatitis C testing, and access to healthcare. The main topics covered in the studies are summarised in Table 2. The most commonly collected information related to drug use and the sharing of injecting equipment, with 16 countries reporting that data were collected on these through behavioural surveillance studies. A total of 14 countries reported collecting information related to HIV, hepatitis B or hepatitis $C$ testing or status, and information related to healthcare usage by IDUs. Information on IDU knowledge and attitudes was collected by only eight countries.

\section{Box 1}

Behavioural indicators among injecting drug users, EU and EFTA countries, reported in 2008 by 8 countries

Eight of the 16 countries with behavioural surveillance studies $^{\text {a }}$ reporting having national indicators.

Countries using each indicator are listed, with the recall period they use (where known).

Sharing needles and/or syringes

- Belgium: not known

- Slovenia: last month and last time

- Switzerland: borrowing and passing on, last month and last six months

- United Kingdom: last month and last six months

Sharing other injecting equipment

- Belgium: not known

- Slovenia: last month and last time

Sharing any injecting equipment

- Finland: last month

- France: borrowing only, last 30 days

- Luxembourg: borrowing only, last 30 days

- Poland: last month, last year, ever

- United Kingdom: last month and last six months

Uptake of voluntary confidential HIV test

- Belgium: last year

- Luxembourg: last five months and ever tested

- Poland: last year and ever tested

- Switzerland: lifetime

- United Kingdom: lifetime

Uptake of voluntary confidential hepatitis C virus test

- Belgium: not known

- Luxembourg: last five months and ever tested

- Poland: last year and ever tested

- Switzerland: lifetime

- United Kingdom: lifetime

Age first injected

- Belgium

- Finland

Condom use

- Finland: last six months (regular or casual partners)

- Luxembourg: last time (by gender)

- Slovenia: last time

- Switzerland: last time, last six months with regular and casual partners

EFTA: European Free Trade Association; EU: European Union.

a Studies using either a repeated survey, cohort or the Treatment Demand Indicator system. 


\section{Behavioural Indicators}

Eight (of 16) countries reported having behavioural indicators related to the monitoring of the impact of programmes to reduce HIV and other infections among IDUs. The seven behavioural indicators that were reported by more than one country, with the countryspecific recall periods used, are shown in Box 1. Three indicators were each reported by five countries: voluntary confidential testing for HIV; voluntary confidential testing for hepatitis $\mathrm{C}$; and the sharing of any injecting equipment in the last month or 30 days There were, however, variations in the recall periods for the testing indicators, with 'ever tested' being used by four countries.

\section{One-off behavioural surveys}

In total, 20 one-off surveys had been used to collect behavioural data in seven countries (France, Ireland, Latvia, Lithuania, Luxembourg, Sweden and United Kingdom; Table 3). In one country, Latvia, these formed a series of surveys that provided data over time; though these surveys had varied methodologically from year to year, they resulted in an informal surveillance structure. In the other countries with multiple one-off surveys, these were not comparable to each other, as they had, for example, recruited IDUs from different areas or had different inclusion criteria. As with the behavioural surveillance studies that used repeated surveys, these one-off surveys had used a wide range of methods and varied in sample size (from 194 to 2,740; mean: 676; median: 463). They also included surveys of prisoners (one survey) and other drug users (one of problem drug users and one of techno events and clubbing population) as well as IDUs. Of the one-off surveys reported, nine had national coverage, two covered only a region or selected regions, eight were local, and for one, the geographical coverage was not given.

Five countries reported both behavioural surveillance studies and one-off surveys. The approaches used for the behavioural surveillance studies in these countries varied: three collected data through repeated surveys and three through their Treatment Demand Indicator systems (one country, France, had used both approaches).

\section{Sampling approaches}

In the absence of a sampling frame for IDUs, all countries had used convenience sampling frameworks to recruit IDUs for one-off surveys or for the repeated surveys used in behavioural surveillance studies (Tables 1 and 3). Most countries used services - typically easy to access (i.e. low-threshold) ones, such as NSPs - as a setting for recruiting and surveying IDUs; however, four countries had used respondent-driven sampling to recruit from communities.

\section{Discussion and conclusion}

Mapping behavioural surveillance in 2008 related to HIV and other STIs among IDUs indicated that 16 countries had conducted behavioural surveillance studies for this subpopulation. A further two countries had undertaken one-off behavioural surveys; and in one of these countries, these surveys resulted in an informal surveillance structure. More countries had behavioural surveillance studies for IDUs than for any of the other population groups: 14 countries for MSM; 13 for the general population; 13 for young people (youth); nine for people living with HIV/AIDS; nine for clients of STI clinics; six for sex workers; and three for migrant populations $[16,17,19]$. A number of these countries have, or have had, more than one behavioural surveillance study among IDUs. Most often the population group with the most studies in a country was also IDUs [16]. While behavioural surveillance related to HIV was more established among IDUs than among other populations, two fifths $(n=10)$ of the 28 countries responding to the survey reported having no behavioural surveillance-related activities among IDUs.

It is important to consider the limitations of our study. The information collected was self-reported and the responses varied greatly in the level of detail provided. The questionnaires were sent to the ECDC national contact person for HIV biological surveillance in each country as there is no specific ECDC contact person for behavioural surveillance. This person may thus have been unaware of the existence of surveys, whether organised or not into a behavioural surveillance system. However, for the questionnaire on behavioural surveillance among IDUs, liaison with the EMCDDA National Focal Point in each EU country and Norway was encouraged. This should have minimised underreporting of existing studies of IDUs. The draft mapping report [16] was also circulated to countries for validation, so providing an opportunity to both make corrections and review its completeness. The data collected here are likely to be robust; however, three countries did not return the questionnaire on behavioural surveillance among IDUs, and two of those that did return the questionnaire and who reported having behavioural surveillance studies among IDUs provided no details. While the response and completion rates were high ( $90 \%$ and $93 \%$, respectively), it cannot be assumed that the non-responding countries and those not providing information are similar to those who did. Our findings should thus be generalised to the whole of the EU/EFTA area cautiously.

The fact that more countries had ongoing behavioural surveillance among IDUs than in the other groups studied might reflect, in part at least, the impact of the EMCDDA-established key indicator on drug-related infectious diseases. Following its inauguration in 1995, EMCDDA set up a standardised system to collect data for this key indicator [3]. This collates the findings from HIV, hepatitis C and hepatitis B prevalence studies among IDUs and has more recently started to collect behavioural data [3]. In response to HIV in the late 1980 s and early 1990s, a number of countries established sero-surveillance studies among IDUs to overcome the potential biases in monitoring HIV prevalence 


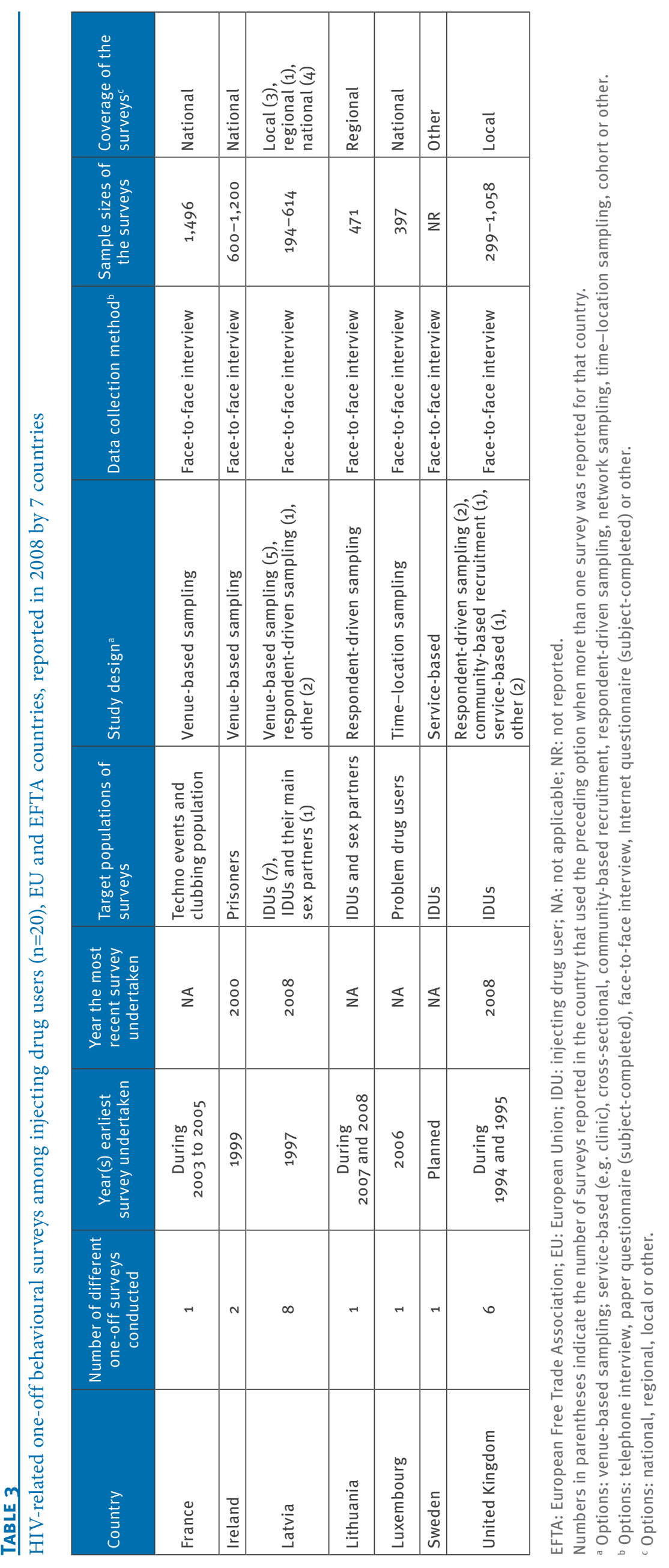


among marginalised populations such as IDUs through diagnostic testing data. These studies, to maximise their public health utility, have also collected behavioural data. Such combined sero-behavioural systems have been established in number of EU Member States over the last 25 years, for example, Spain (in Catalonia) [20], Estonia [21] and United Kingdom (England, Wales and Northern Ireland) [22]. Through its drug-related infectious diseases key indicator, the EMCDDA has encouraged the maintenance and continued development of such studies across the EU.

Undertaking surveys among IDUs presents a number of substantial practical difficulties. In particular, due to the illicit nature of drug injecting and the high levels of marginalisation and associated stigma, accessing populations who inject drugs can be difficult, and there is, of course, no population-based sampling frame. Thus surveys of IDUs typically use accessibility sampling approaches $[11,23]$, either to access individuals in the community or through the services provided to them. This need to use convenience sampling approaches is reflected in the range of methods used to collect behavioural data. These approaches ranged from collecting data from the clients of addiction treatment services using the Treatment Demand Indicator system, through the purposive sampling of individuals in contact with services provided to drug users (such as NSPS, OST, drop-in centres and outreach), to community-based recruitment, including the use of respondent-driven sampling [23]. Sampling through specialist services for drug users (such as services providing NSPs and OST) was the most widely used approach, probably reflecting the extensive provision of a range of such services in many European countries [4].

In most countries with behavioural surveillance studies of IDUs, these were being conducted annually or at regular intervals, indicating that these systems were probably routine surveillance activities. Routine surveillance of risk among IDUs is important, considering the potential for HIV to spread very rapidly through injecting drug use [11]. The samples sizes used in the surveys varied greatly, with the largest samples being about 30 times larger than the smallest. However, in part this variation will reflect the different population sizes of the countries and also what is known about the extent of injecting drug use in each country. It is likely that the range of sampling approaches used reflects what is appropriate, considering the local epidemics of drug use and the responses to these and, of course, the resources available for surveillance in each country. The systems thus took a range of forms, used a variety of recruitment approaches and settings, and varied greatly in size. These variations probably reflect a wide range in the quality, robustness and sustainability of the systems, although these cannot be objectively assessed through a mapping exercise of this kind.

Examination of the topics covered in the behavioural surveillance studies among IDUs indicates that a wide range of topics were addressed. The main ones (reported in at least two thirds of the countries with behavioural surveillance studies) concerned drug use, injecting risks, HIV and hepatitis C testing, hepatitis B vaccination and sexual risks. This list of topics is not surprising considering the ease with which HIV and hepatitis $B$ and $C$ viruses can be transmitted through unsafe injecting practices, but the lack of sexual risk information in a third of the countries is of concern, given that STIS, HIV and hepatitis B virus are readily transmitted through unprotected sexual intercourse.

Almost half of the countries with behavioural surveillance studies had identified key behavioural indicators that they specifically used for monitoring purposes. The most common key indicators focused on voluntary confidential testing for HIV and hepatitis C, and the sharing of injecting equipment. Half of the countries with key indicators had included condom use as indicator. The set of indicators suggested by ECDC after consultation in the 2009 expert meeting [16] are shown in Box 2.

These indicators include those that are most frequently used in the eight countries with key indicators (i.e. testing for HIV, testing for hepatitis $\mathrm{C}$ virus and sharing injecting equipment) and they also reflect the

\section{Box 2}

Suggested indicators for use with injecting drug users, EU and EFTA countries

Transversal indicators (those common with other population groups) ${ }^{a}$

Main indicators:

- condom use at last sexual intercourse ${ }^{b}$

- HIV testing and test result (reported or measured) ${ }^{\mathrm{b}}$

Also where appropriate:

- number of sexual partners in the last 12 months

- involvement in sex work (as client)

Suggested IDU-specific indicators

Main indicators:

- needles and syringe sharing ${ }^{\mathrm{b}, \mathrm{c}}$

- injecting frequency ${ }^{\mathrm{b}, \mathrm{c}}$

- number of new needles/syringes obtained $d^{\mathrm{b}, \mathrm{c}}$

- recently received a substitute drug ${ }^{\mathrm{b}, \mathrm{c}}$

Additional indicators:

- hepatitis C testing (same format as for HIV testing transversal indicator) ${ }^{a}$

- years since first injected ${ }^{\mathrm{b}}$

- having been paid for sex ${ }^{b}$

Other possible options include:

- number of sharing partners ${ }^{\mathrm{b}, \mathrm{c}}$

- ever injected in prison

EFTA: European Free Trade Association; EU: European Union.

Source: [16].

Indicators for which the European Monitoring Centre for Drugs and Drug Addiction (EMCDDA) is collecting behavioural data. Recall periods for these indicators need to be agreed, although the mapping exercise indicates that the last month (last 28 or 30 days) is commonly used for these, and would probably be an appropriate period where injecting is a regular event (e.g. from several times a week to daily), but may be too short where injecting is less frequent. 
topics covered in most behavioural surveillance studies. While further consultation is needed on the recall periods and the specific definitions for some of these indicators, the studies do provide a framework from which a core set of behavioural indicators for IDUs could be established. The adoption of a core set of indicators, and their incorporation in national behavioural surveillance studies for IDUs, would then allow comparisons of behavioural surveillance data across countries. This currently cannot be done robustly due to the wide range of different indicators being used across the EU and EFTA.

Behavioural surveillance was, in 2008, more frequently reported among IDUs than in other subpopulations (followed closely by MSM, general population and youth) [16]; however, 10 of the 28 of the countries responding reported no behavioural surveillance among IDUs. The approach used here, a mapping survey, may have resulted in under-reporting of surveys, particularly as not all countries replied, and so the findings should be treated cautiously. Even so, the diversity of indicators found indicates a need to harmonise behavioural surveillance indicators among IDUs across European countries, and this should consider international guidance [24] when developing any indicators. To this end, EMCDDA, in consultation with ECDC and international experts, is currently finalising its protocol for collecting data, including behavioural data, on drug-related infectious diseases among IDUs.

\section{The ECDC HIV and STI Behavioural Surveillance}

\section{Mapping Group}

The full report (ECDC Technical Report Mapping of HIV/STI behavioural surveillance in Europe [16]) was commissioned by ECDC, coordinated by Marita van de Laar and produced by the Institute for Social and Preventive Medicine (IUMSP), University of Lausanne, Switzerland, working with an international team of experts listed below. The main role of each person is included in parentheses; each expert focused on one population group.

Françoise Dubois-Arber, Institute for Social and Preventive Medicine (IUMSP), Lausanne, Switzerland (team leader, youth); Brenda Spencer, IUMSP, Lausanne, Switzerland (general population); Vivian Hope, London School of Hygiene and Tropical Medicine, United Kingdom (IDUs); Jonathan Elford, City University, London, United Kingdom (MSM); France Lert, Institut national de la santé et de la recherché médicale, France (people living with HIV/AIDS); Helen Ward, Imperial College, London, United Kingdom (sex workers); Nicola Low, Institute for Social and Preventive Medicine, Berne, Switzerland (STI clinic patients); Mary Haour-Knipe, freelance consultant, formerly with the International Organization for Migration (migrants and ethnic minorities); André Jeannin, IUMSP, Lausanne, Switzerland (organisation of survey); JeanPierre Gervasoni, IUMSP, Lausanne, Switzerland (organisation of survey); Marie-Jeanne Pellaz, IUMSP, Lausanne, Switzerland (secretarial assistance); Bertrand Graz, IUMSP, Lausanne, Switzerland (literature review); Marita van de Laar, ECDC, Stockholm, Sweden (coordinator).
References

1. Wiessing L, Ncube F, Hedrich D, Griffiths P, Hope V, Gill N, et al. Surveillance of infectious diseases in IDUs across the EU: information from the EU expert network. Euro Surveill. 2004;8(4):pii=2368. Available from: http://www. eurosurveillance.org/ViewArticle.aspx?Articleld $=2368$

2. Health Protection Agency, Health Protection Scotland, National Public Health Service for Wales, CDSC Northern Ireland, CRDHB. Shooting up: infections among injecting drug users in the United Kingdom 2008. London: Health Protection Agency; October 2009. Available from: http://www.hpa.org.uk/web/ HPAwebFile/HPAweb_C/1254510653792

3. European Monitoring Centre for Drugs and Drug Addiction (EMCDDA). Drug-related infectious diseases. Lisbon: EMCDDA. [Accessed 9 Jul 2010]. Available from: http://www.emcdda. europa.eu/themes/key-indicators/drid

4. European Monitoring Centre for Drugs and Drug Addiction (EMCDDA). Trends in injecting drug use in Europe. Selected Issue 2010. Lisbon: EMCDDA: 2010. Available from: http:// www.emcdda.europa.eu/publications/selected-issues/ injecting

5. Hedrich D, Pirona A. Wiessing L. From margin to mainstream: the evolution of harm reduction responses to problem drug use in Europe. Drugs: Education, Prevention, and Policy. 2008;15(6):503-17. Available from: http://informahealthcare. com/doi/abs/10.1080/09687630802227673

6. Wodak A, Cooney A. Do needle syringe programs reduce HIV infection among injecting drug users: a comprehensive review of the international evidence. Subst Use Misuse. 2006;41(6-7):777-813.

7. World Health Organization (WHO). Effectiveness of sterile needle and syringe programming in reducing HIV/AIDS among injecting drug users. (Evidence for action technical papers). Geneva: WHO; 2004. Available from: http://www.who.int/hiv/ pub/prev_care/effectivenesssterileneedle.pdf

8. Vickerman P, Hickman M, Judd A. Modelling the impact on hepatitis $C$ transmission of reducing syringe sharing: London case study. Int J Epidemiol. 2007:36(2):396-405.

9. Van Den Berg C, Smit C, Van Brussel G, Coutinho R, Prins $M$; Amsterdam Cohort. Full participation in harm reduction programmes is associated with decreased risk for human immunodeficiency virus and hepatitis C virus: evidence from the Amsterdam Cohort Studies among drug users. Addiction. 2007;102(9):1454-62.

10. Hope VD, Hickman M, Ngui SL, Jones S, Telfer M, Bizzarri $M$, et al Measuring the incidence, prevalence and genetic relatedness of hepatitis $C$ infections among a community recruited sample of injecting drug users, using dried blood spots. J Viral Hepat. 2011;18(4):262-70.

11. Des Jarlais DC, Dehne K, Casabona J. HIV surveillance among injecting drug users. AIDS. 2001;15 Supplement 3:S13-22.

12. Joint United Nations Programme on HIV/AIDS (UNAIDS), World Health Organization (WHO). UNAIDS/WHO Working Group on Global HIV/AIDS and STI Surveillance. Guidelines for second generation HIV surveillance. Geneva: UNAIDS and WHO; 2000. WHO/CDS/CSR/EDC/2000; UNAIDS/00.03E. Available from: http://data.unaids.org/publications/irc-pubo1/ jc370-2ndgeneration en.pdf

13. European Monitoring Centre for Drugs and Drug Addiction (EMCDDA). An overview of the drug-related infectious diseases (DRID) key indicator. Lisbon: EMCDDA; 2009. Available from: http://www.emcdda.europa.eu/publications/methods/ drid-overview

14. European Centre for the Epidemiological Monitoring of AIDS (EuroHIV). HIV/AIDS surveillance in Europe. Mid-year report 2001. No. 65. Saint-Maurice: Institut de Veille Sanitaire; 2001. Available from: http://www.ecdc.europa.eu/en/activities/ surveillance/hiv/Documents/report_eurohiv_midyear_2001.pdf

15. European Centre for the Epidemiological Monitoring of AIDS (EuroHIV). EuroHIV 2006 survey on HIV and AIDS surveillance in the WHO European Region. Saint-Maurice: Institut de veille sanitaire; 2007. Available from: http://www.invs.sante.fr/ publications/2007/eurohiv_2006/HIV_AIDS_Web.pdf

16. European Centre for Disease Prevention and Control. Mapping of HIV/STI behavioural surveillance in Europe. ECDC Technical Report. Stockholm: ECDC; September 2009. Available from: http://www.ecdc.europa.eu/en/publications/ Publications/0909_TER_Mapping_of_HIV_STI_Behavioural Surveillance_in_Europe.pdf

17. Dubois-Arber F, Jeannin A, Spencer B, Gervasoni JP, Graz B, Elford I, et al. Mapping HIV/STI behavioural surveillance in Europe. BMC Infect Dis. 2010;10:290. 
18. European Monitoring Centre for Drugs and Drug Addiction (EMCDDA). An overview of the treatment demand key indicator (TDI). Lisbon: EMCDDA; 2009. Available from: http://www. emcdda.europa.eu/publications/methods/tdi-overview

19. Elford J, Jeannin A, Spencer B, Gervasoni JP, van de Laar MJ, Dubois-Arber F; et al.. HIV and STI behavioural surveillance among men who have sex with men in Europe. Euro Surveill. 2009;14(47): pii=19414. Available from: http://www.

eurosurveillance.org/ViewArticle.aspx?Articleld=19414

20. Centre d'Estudis Epidemiològics sobre les Infeccions de Transmissió Sexual i Sida de Catalunya (CEEISCAT). SIVES 2008 : integrated Aids/HIV/STI surveillance system of Catalonia (SIVES): biennial epidemiological report . Barcelona: Generalitat de Catalunya, Departament de Salut; 2008. (Technical document CEEISCAT, 19). Available from: http:// www.ceescat.org/Documents_Ing1.htm

21. Vorobjov S, Uusküla A, Abel-Ollo K, Talu A, Rüütel K, Des Jarlais DC. Comparison of injecting drug users who obtain syringes from pharmacies and syringe exchange programs in Tallinn, Estonia. Harm Reduct J. 2009;6:3.

22. Hope VD, Judd A, Hickman M, Sutton A, Stimson GV, Parry JV, et al. HIV prevalence among injecting drug users in England and Wales 1990 to 2003: evidence for increased transmission in recent years. AIDS. 2005;19(11):1207-14.

23. Magnani R, Sabin K, Saidel T, Heckathorn D. Review of sampling hard-to-reach and hidden populations for HIV surveillance. AIDS. 2005;19 Suppl 2:S67-72.

24. World Health Organization (WHO). WHO, UNODC, UNAIDS technical guide for countries to set targets for universal access to HIV prevention, treatment and care for injecting drug users. Geneva: WHO; 2009. Available from: http://www.unodc.org/ documents/hiv-aids/idu_target_setting_guide.pdf 